\section{BDNF and episodic memory in patients with bipolar disorder}

\section{BDNF e memória episódica em pacientes com transtorno afetivo bipolar}

\section{Dear Editor,}

Brain-derived neurotrophic factor (BDNF), a member of the neurotrophin family, has a whole brain distribution that is highly expressed in key cerebral structures related to psychiatric disorders and cognition such as the amygdala, pre-frontal cortex hypothalamus and hippocampus. ${ }^{1}$ The most widely studied polymorphism is Val66Met, which impairs BDNF trafficking and secretion and, according to some studies, Met carriers are more prone to exhibit memory and learning impairments. ${ }^{1,2}$

Recently, the Revista Brasileira de Psiquiatria published a well designed and interesting association study by Tramontina et al. that reported results on Val66Met BDNF polymorphism and performance on the Wisconsin Card Sorting Test in 64 patients with bipolar disorder. ${ }^{3}$ The authors found that the percentage of non-perseverative errors was significantly higher in Val/Val carriers, thus contradicting previous findings on cognitive impairment in Met allele carriers. ${ }^{1,2}$

Considering the relationship between BDNF and processes of memory and learning, as well as the existence of episodic deficits in bipolar patients even when euthymic, ${ }^{4,5}$ we launched an investigation into the relationship between the Val66Met polymorphism and the performance in verbal and visual episodic memory tasks (COEP UFMG process approval number 018/09).
In the initial phase of the study, 63 bipolar disorder subjects in the euthymic phase (as described in Moreira et al. ${ }^{5}$ ), diagnosed according to the DSM IV using a structured interview, underwent the Auditory Verbal Learning Test (RAVLT) and the Rey Complex Figure Test (RCFT). RAVLT measures verbal learning, retention of information and recognition memory. It also measures susceptibility to both proactive and retroactive interference, which is related to attention and executive functions. RCFT measures visio-construction and visual memory. After genotyping, the subjects were grouped according to the presence or absence of Met allele (Val/Val, $\mathrm{n}=52 ; \mathrm{Val} / \mathrm{Met}+\mathrm{Met} / \mathrm{Met}, \mathrm{n}=11)$. The two groups were compared using the Mann-Whitney test (or Chisquare test for categorical variables) and results were considered statistically significant when $\mathrm{p}<0.05$. We did not find significant differences between the two groups on measures of verbal and visual episodic memory (Table 1). Nevertheless, individuals in the $\mathrm{Val} / \mathrm{Met}+\mathrm{Met} / \mathrm{Met}$ group performed significantly worse than individuals in the $\mathrm{Val} / \mathrm{Val}$ group relative to measurements of susceptibility to proactive interference $(z=2.923 ; \mathrm{p}=0.003)$.

Our results reinforce the existence of worse cognitive functioning and greater difficulty in terms of proactive interference susceptibility in Met-allele carriers in particular. It must be emphasized that proactive interference susceptibility is not only related to memory and learning, but also to attention and executive functions. Thus, our initial results are partly different from those found by Tramontina et al. These differences could be attributed to sample characteristics since bipolar disorder is a heterogeneous clinical condition and certain patient subgroups may exhibit more severe cognitive impairments than others. ${ }^{3}$

Certain limitations of this study must be noted. The sample is small and all subjects were using pharmacological therapy during the study. Future studies with larger samples are needed to evaluate the hypothesis that individuals carrying the Met allele have greater difficulty in cognitive processes of memory and executive functions.

Table 1 - Comparison between VAL/VAL AND VAL/MET + MET/MET groups in sociodemographic and neuropsychological measures

\begin{tabular}{|c|c|c|c|c|c|c|c|c|c|c|}
\hline & \multicolumn{8}{|c|}{ BDNF val66met polimorphism } & \multirow[b]{2}{*}{$x^{2}$ or $z$} & \multirow[b]{2}{*}{$\mathbf{p}$} \\
\hline & Mean & $\begin{array}{l}\text { Standard } \\
\text { Deviation }\end{array}$ & $\mathbf{N}$ & $\%$ & Mean & $\begin{array}{l}\text { Standard } \\
\text { Deviation }\end{array}$ & $\mathbf{N}$ & $\%$ & & \\
\hline Age & 48.73 & 13.36 & & & 40.90 & 10.97 & & & 1.595 & 0.111 \\
\hline Gender (Female) & & & 9 & 82 & & & 38 & 73 & 0.366 & 0.545 \\
\hline RAVLT A1 & 5.36 & 0.92 & & & 5.06 & 1.51 & & & 0.852 & 0.394 \\
\hline $\begin{array}{l}\text { RAVLT Proactive } \\
\text { interference }\end{array}$ & 0.00 & 0.00 & & & 0.52 & 0.61 & & & 2.923 & 0.003 \\
\hline $\begin{array}{l}\text { RAVLT Retroactive } \\
\text { Interference }\end{array}$ & 0.45 & 0.69 & & & 0.31 & 0.54 & & & 0.689 & 0.491 \\
\hline RAVLT Forget Speed & 0.73 & 0.47 & & & 0.54 & 0.50 & & & 1.140 & 0.254 \\
\hline
\end{tabular}


Lafaiete Moreira

Laboratórios Integrados de Neuropsicologia (LINEU), Universidade Federal de Minas Gerais (UFMG), Brazil

Programa de Pós Graduação em Medicina Molecular, Faculdade de Medicina, Universidade Federal de Minas Gerais (UFMG), Belo Horizonte, MG, Brazil

Fernando Silva Neves Departamento de Saúde Mental, Faculdade de Medicina, Universidade Federal de Minas Gerais (UFMG), Belo Horizonte, MG, Brazil

Programa de Pós-Graduação em Neurociências, Universidade Federal de Minas Gerais (UFMG), Belo Horizonte, MG, Brazil

Instituto Nacional de Ciência e Tecnologia de Medicina Molecular, Faculdade de Medicina, Universidade Federal de

Minas Gerais (UFMG), Belo Horizonte, MG, Brazil

Marco Aurélio Romano-Silva

Departamento de Saúde Mental, Faculdade de Medicina, Universidade Federal de Minas Gerais (UFMG), Belo Horizonte, MG, Brazil

Programa de Pós-Graduação em Neurociências, Universidade Federal de Minas Gerais (UFMG), Belo Horizonte, MG, Brazil

Programa de Pós-Graduação em Medicina Molecular,

Universidade Federal de Minas Gerais (UFMG), Belo Horizonte, MG, Brazil

Instituto Nacional de Ciência e Tecnologia em Medicina Molecular, Faculdade de Medicina, Universidade Federal de Minas Gerais (UFMG), Belo Horizonte, MG, Brazil
Leandro Fernandes Malloy-Diniz

Laboratórios Integrados de Neuropsicologia (LINEU),

Universidade Federal de Minas Gerais (UFMG), Brazil Programa de Pós-Graduação em Neurociências, Universidade Federal de Minas Gerais (UFMG), Belo Horizonte, MG, Brazil

Programa de Pós-Graduação em Medicina Molecular, Universidade Federal de Minas Gerais (UFMG),

Belo Horizonte, MG, Brazil

Instituto Nacional de Ciência e Tecnologia em Medicina Molecular, Faculdade de Medicina, Universidade Federal de Minas Gerais (UFMG), Belo Horizonte, MG, Brazil

Humberto Corrêa

Departamento de Saúde Mental, Faculdade de Medicina, Universidade Federal de Minas Gerais (UFMG), Belo Horizonte, MG, Brazil

Programa de Pós-Graduação em Neurociências, Universidade Federal de Minas Gerais (UFMG), Belo Horizonte, MG, Brazil Programa de Pós-Graduação em Medicina Molecular, Universidade Federal de Minas Gerais (UFMG), Belo Horizonte, MG, Brazil

Instituto Nacional de Ciência e Tecnologia em Medicina Molecular, Faculdade de Medicina, Universidade Federal de Minas Gerais (UFMG), Belo Horizonte, MG, Brazil Hopital Sainte Anne. Université Paris-Decartes, Paris, France.

\section{Disclosures}

\begin{tabular}{|c|c|c|c|c|c|c|c|}
\hline $\begin{array}{l}\text { Writing group } \\
\text { member }\end{array}$ & Employment & $\begin{array}{l}\text { Research } \\
\text { grant }^{1}\end{array}$ & $\begin{array}{l}\text { Other research grant } \\
\text { or medical continuous } \\
\text { education }\end{array}$ & $\begin{array}{l}\text { Speaker's } \\
\text { honoraria }\end{array}$ & $\begin{array}{l}\text { Ownership } \\
\text { interest }\end{array}$ & $\begin{array}{l}\text { Consultant/ } \\
\text { Advisory } \\
\text { board }\end{array}$ & Other $^{3}$ \\
\hline Lafaiete Moreira & LINEU & - & - & - & - & - & - \\
\hline $\begin{array}{l}\text { Fernando Silva } \\
\text { Neves }\end{array}$ & UFMG & & & & & & \\
\hline $\begin{array}{l}\text { Marco Aurélio } \\
\text { Romano-Silva }\end{array}$ & UFMG & - & - & - & - & - & - \\
\hline $\begin{array}{l}\text { Leandro Fernandes } \\
\text { Malloy-Diniz }\end{array}$ & $\begin{array}{l}\text { LINEU } \\
\text { UFMG }\end{array}$ & & & & & & \\
\hline Humberto Corrêa & $\begin{array}{c}\text { UFMG } \\
\text { Hopital Sainte Anne }\end{array}$ & - & - & - & - & - & - \\
\hline
\end{tabular}

* Modest

** Significant

*** Significant: Amounts given to the author's institution or to a colleague for research in which the author has participation, not directly to the authol Note: LINEU = Laboratórios Integrados de Neuropsicologia; UFMG = Universidade Federal de Minas Gerais.

For more information, see Instructions for Authors.

References

1. Murer MG, Yan Q. Raisman-Vozari, R. Brain-derived neurotrophic factor in the control brain, and in Alzheimer's disease and Parkinson's disease. Prog Neurobiol. 2001;63(1):71-124.

2. Egan MF, Kojima M, Callicott JH, Goldberg TE, Kolachana BS, Bertolino A, Zaitsev E, Gold B, Goldman D, Dean M, Lu B, Weinberger DR.. The BDNF val66met polymorphism affects activity-dependent secretion of BDNF and human memory and hippocampal function. Cell. 2003;112(2):257-69.

3. Tramontina JF, Yates D, Magalhães PVS, Trentini C, Sant'Anna MK, Fries GR, Bock H, Saraiva-Pereira ML, Kapczinski F. Brain-derived neurotrophic factor gene val66met polymorphism and executive funcioning in patients with bipolar disorder. Rev Bras Psiquiatr. 2009;31(2):136-40.

4. Martínez-Arán A, Vieta E, Reinares M, Colom F, Torrent C, Sánchez-Moreno J, Benabarre A, Goikolea JM, Comes M, Salamero M. Cognitive function across manic or hypomanic, depressed, and euthymic states in bipolar disorder. Am J Psychiatry. 2004;161(2):262-70.

5. Moreira L, Neves FS, Schlottfeldt CG, Abrantes SSC, Moraes PHP, RomanoSilva MA, Correa H, Malloy-Diniz LF. Visual and verbal memory in euthymic bipolar patients: Impacts of subtype, psychotic symptoms and suicide behavior. Clin Neuropsychiatry. 2010. Ahead publication. [cited 2010 June 28]. Available from: http://www.clinicalneuropsychiatry.org/. 